\title{
Capacidad operativa de la gestión de desechos sólidos en el cantón Gonzalo Pizarro, Ecuador
}

\author{
Operational capacity of solid waste management in Gonzalo Pizarro canton, Ecuador \\ Capacidade operacional de gestão de resíduos sólidos no cantão Gonzalo Pizarro, Equador
}

\section{Juan Pablo Morales Corozo}

j.p.shevarojo@gmail.com

Universidad Nacional Experimental de los Llanos Occidentales "Ezequiel Zamora" https://orcid.org/0000-0002-4538-4488

\section{RESUMEN}

Las empresas se encuentran en constante competencia y la mejora en el desempeño económico motivo por el cual están influenciados por sus capacidades operativas. El estudio de la capacidad operativa es importante y cobra cada vez más relevancia dado que las empresas e industrias se encuentran realizando procesos de mejoramiento continuo, utilizando al máximo sus recursos con el propósito de conservar su permanencia en el mercado. La gestión ambiental dentro de los gobiernos autónomos descentralizados municipales comprende varias actividades encaminadas conservar el ambiente y proponer tareas para la conservación, siendo muy importante conocer la capacidad operativa, teniendo en cuenta las competencias establecidas en los diferentes instrumentos legales. El objetivo de la presente investigación es establecer una herramienta técnica sobre la cual se planifique el Plan Operativo Anual y el Plan Anual de Compras en la Dirección de Gestión de Ambiente, para desempeñar las funciones en cuanto a cada una de las actividades ambientales de acuerdo a las competencias exclusivas en materia de gestión integral de los desechos sólidos. De conformidad al análisis realizado se tiene que la capacidad real de la gestión integral de los desechos sólidos es de 58.862 h/año, teniendo una eficiencia de $65,21 \%$, esto es debido a variables de causas asignables que se debe tomar en cuenta (trabajos múltiples, paradas continuas de maquinaria por mantenimiento correctivo, actividades operativas imprevistas), que no tienen nada que ver con la operatividad y la planificación.

Palabras clave: gestión de desechos sólidos, capacidad operativa, gestión administrativa, objetivos estratégicos

\begin{abstract}
Companies are in constant competition and improvement in economic performance, which is why they are influenced by their operational capabilities. The study of operational capacity is important and becomes increasingly relevant given that companies and industries are carrying out continuous improvement processes, using their resources to the maximum in order to maintain their permanence in the market. Environmental management within the municipal decentralized autonomous governments includes several activities aimed at conserving the environment and proposing tasks for conservation, being very important to know the operational capacity, taking into account the powers established in the different legal instruments. The objective of this research is to establish a technical tool on which the Annual Operating Plan and the Annual Purchasing Plan are planned in the Environment Management Department, to perform the functions regarding each of the environmental activities according to the exclusive competencies in terms of comprehensive management of solid waste. In accordance with the analysis carried out, the real capacity of the integral management of solid waste is $58,862 \mathrm{~h} /$ year, with an efficiency of $65.21 \%$, this is due to variables of assignable causes that must be taken into account ( multiple jobs, continuous stoppages of machinery for corrective maintenance, unforeseen operational activities), which have nothing to do with operability and planning.
\end{abstract}

Keywords: Solid waste management, operational capacity, administrative management, strategic objectives.

\section{RESUMO}

As empresas estão em constante competição e melhoria no desempenho econômico, razão pela qual são influenciadas por suas capacidades operacionais. O estudo da capacidade operacional é importante e se torna cada vez mais relevante, visto que empresas e indústrias estão realizando processos de melhoria contínua, utilizando ao máximo seus recursos para manter sua permanência no mercado. A gestão ambiental nas autarquias autónomas descentralizadas municipais inclui diversas actividades destinadas a conservar o ambiente e propor tarefas para a conservação, sendo muito importante conhecer a capacidade operacional, tendo em conta as competências estabelecidas nos diferentes instrumentos legais. O objetivo desta pesquisa é estabelecer uma ferramenta técnica sobre a qual o Plano Operacional Anual e o Plano Anual de Compras são planejados no Departamento de Gestão Ambiental, para desempenhar as funções relativas a cada uma das atividades ambientais de acordo com as competências exclusivas em termos de gestão integral de resíduos sólidos. De acordo com a análise realizada, a capacidade real da gestão integral de resíduos sólidos é de 58.862 h/ano, com uma eficiência de $65,21 \%$, isso se deve a variáveis de causas atribuíveis que devem ser levadas 
em consideração (trabalhos múltiplos, paradas de máquinas para manutenção corretiva, atividades operacionais imprevistas), que nada têm a ver com operacionalidade e planejamento.

Palavras-chave: gestão de resíduos sólidos, capacidade operacional, gestão administrativa, objetivos estratégicos

\section{INTRODUCCIÓN}

Las empresas se encuentran en constante competencia y la mejora en el desempeño económico motivo por el cual están influenciados por sus capacidades operativas, "debido a que las organizaciones son consideradas como una gran red de rutinas en sus diferentes procesos de producción, el monitoreo del desempeño de las distintas rutinas del negocio, el seguimiento de las actividades de control" (Hernández, et. al., 2017, p. 18). El estudio de la capacidad operativa es importante y cobra cada vez más relevancia dado que las empresas e industrias se encuentran realizando procesos de mejoramiento continuo, utilizando al máximo sus recursos con el propósito de conservar su permanencia en el mercado. La gestión administrativa en las empresas públicas se encuentra variando de manera considerable obligando a instituir criterios técnicos más acertados para la búsqueda de mejores resultados de acuerdo a los requerimientos legales establecidos por los organismos de control. Kogut y Zander, (1992) sostiene que parte de la innovación es la generación de conocimiento siento cada vez más las empresas e instituciones públicas las que acceden a generar más activos intangibles, "ya sea bajo innovación abierta o interactiva" (Ritala \& Blomqvist, 2009, p. 572). La innovación aplicada a los modelos de gestión son considerados retos estratégicos en cuanto a su aplicabilidad.

La gestión ambiental dentro de los gobiernos autónomos descentralizados municipales comprende varias actividades encaminadas conservar el ambiente y proponer tareas para la conservación, siendo muy importante conocer la capacidad operativa, teniendo en cuenta las competencias establecidas en los diferentes instrumentos legales. El Gobierno Autónomo Descentralizado Municipal del cantón Gonzalo Pizarro de acuerdo a lo manifestado en el Código Orgánico de Ordenamiento Territorial, Autonomía y Descentralización el propósito prioritario es garantizar un ambiente sano y libre de contaminación, interviniendo en actividades de control, regularización y prevención de daños ambientales contribuyendo al desarrollo social y económico del municipio de Gonzalo Pizarro. De acuerdo a la manifestado anteriormente se realizar varias actividades operativas, técnicas y administrativas a beneficio de los habitantes que se encuentran dentro de la circunscripción territorial, cuyo cumplimiento de las metas depende de sobremanera de contar con todos los recursos necesarios e indispensables, siendo necesario la realización de modelos de gestión administrativa, contribuyendo a la generación eficiente de resultados en materia ambiental.

Para tal efecto y llevando concordancia con el Plan de Desarrollo y Ordenamiento Territorial se plantean los siguientes objetivos:

- Propiciar la conservación ambiental, estableciendo como principio la corresponsabilidad ciudadana en el manejo sostenible de los recursos naturales, mediante políticas, normas, instrumentos y acciones de desarrollo que permitan la recuperación de espacios naturales degradados.

- Impulsar de manera sistema la resiliencia en el territorio para enfrentar amenazas de origen natural y antrópico, mediante acciones que propendan una adecuada gestión del riesgo de desastres.

La finalidad de estos objetivos conlleva a la propuesta de alternativas para manejar adecuadamente la gestión con los recursos necesarios que permitan contar con la cantidad y calidad apropiada de materiales, herramientas, equipos y personal para incrementar la capacidad operativa 
de la Dirección de Gestión de Riesgos del Gobierno Autónomo Descentralizado Municipal del cantón Gonzalo Pizarro.

El objetivo de la presente investigación es establecer una herramienta técnica sobre la cual se planifique el Plan Operativo Anual y el Plan Anual de Compras en la Dirección de Gestión de Ambiente, para desempeñar las funciones en cuanto a cada una de las actividades ambientales de acuerdo a las competencias exclusivas en materia de gestión integral de los desechos sólidos.

\section{MARCO TEÓRICO}

La Administración de operaciones comprende la agrupación de actividades que crean valor en forma de servicios o bienes transformando los insumos en productos terminados. "Es la función que permite a las organizaciones alcanzar sus metas mediante adquisición y utilización de recursos" (Montes, 2014, p. 4). La administración de operaciones en un inicio era utilizada en las fábricas y empresas manufactureras. En la actualidad permite a cualquier organización pública o privada controlar los procesos y transformar los insumos en bienes y servicios. En las empresas públicas no se crea bienes tangibles, sino servicios por lo cual la situación de la producción es menos evidente.

Las principales funciones de administración de operaciones en los gobiernos municipales se encuentran relacionadas con las actividades productivas, destacándose los siguientes:

- Diseño de los servicios a partir de las competencias a partir del Plan de Desarrollo y Ordenamiento Territorial, donde se planifica el proceso productivo y del servicio público.

- Diseño de los procesos productivos definiendo la tecnología a utilizar para mejorar los servicios, el personal necesario a contratar, y el control de los procesos.

- Tener una correcta gestión de inventarios en coordinación con los responsables de compras públicas definiendo los bienes y servicios necesarios para brindar los servicios con base a sus competencias.

- Considerar la importancia de la calidad de los servicios y el mantenimiento como procesos auxiliares necesarios en la producción, siendo el primero el que ayuda a definir los estándares de calidad del servicio, mientras que el segundo revisa y repara la maquinaria y herramientas utilizadas para brindar el servicio.

- La coordinación con los departamentos financieros las tasas de cobro de los servicios prestados mediante los diferentes instrumentos legales, a la vez gestiona los procesos de pago a los proveedores.

"Un sistema de operaciones es algo complejo, ya que en el concurren varias disciplinas tecnológicas, donde se ven reflejadas las actitudes y filosofías de los directores, así como la influencia de los clientes y proveedores" (Córdova, 2019, p. 16). Contar con una administración de operaciones bien definida en los gobiernos municipales permite una interacción con los usuarios o clientes, distribuidores, contratistas e instituciones públicas. De esta manera, la producción no finaliza cuando se brinda el servicio ya que el sistema de adquisición y venta del servicio son la parte integral del proceso. 


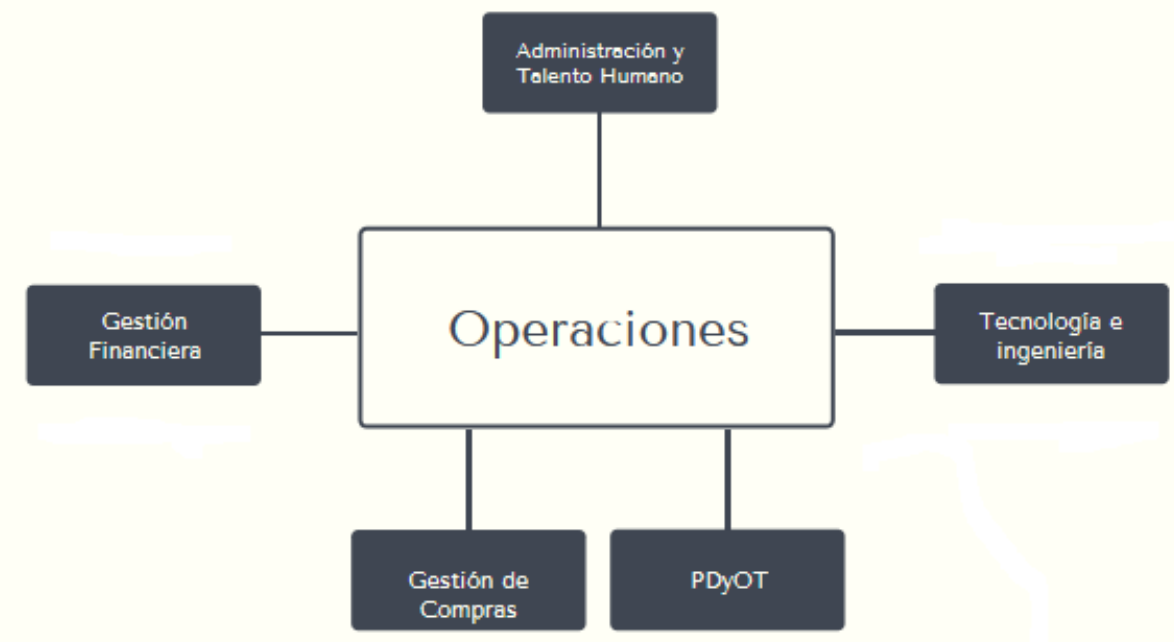

Fuente: (Morales, 2022)

La estrategia de las operaciones proporciona un insumo para la comprensión de "las relaciones internas de operaciones y para ver las conexiones entre operaciones y otras áreas" (Córdova, 2019, p. 20). La importancia de la administración de operaciones en los gobiernos municipales va encaminada a la búsqueda del cumplimiento de las competencias que permita armonizar los procesos internos de tal manera que se cumpla con los objetivos trazados en el Plan de Desarrollo y Ordenamiento Territorial, corrigiendo la falta de planificación y criterios en las diferentes áreas.

Al momento de la formulación de los objetivos institucionales como gobierno municipal los sistemas de producción empiezan a dar forma eligiendo el producto que se pretende brindar a los usuarios. "El producto necesita de un procedimiento específico, el cual debe ser lo más económico posible, teniendo en cuanta la capacidad de producción" (Montes, 2014, p. 11). La capacidad depende de manera esencial del talento humano, materiales, herramientas y recursos financieros designados por el gobierno central y los ingresos por concepto de pago de servicios. Montes, (2014) dentro de su investigación sostiene que la "capacidad debe permitir el logro del objetivo a un plazo más o menos largo, el cual se fija al inicio de la operación" (p. 11). El éxito o el fracaso del cumplimiento de los objetivos institucionales depende de la toma de decisiones a partir del análisis detallado de la operatividad y la concepción del sistema productivo en cuanto al cumplimiento de las competencias exclusivas.

La capacidad de mejora en los gobiernos municipales comprende la agrupación de habilidades, procesos y operaciones encaminados a mejorar radicalmente los "procesos existentes de operaciones o para crear e implementar nuevos y únicos procesos de operaciones" (Hernández, et. al., 2017, p. 19). La mejora operativa es aplicable generalmente a los procesos operativos buscando nuevas formas de hacer el trabajo más eficiente atendiendo a los usuarios de la mejor manera.

\section{MATERIALES Y MÉTODOS}

El cantón Gonzalo Pizarro, es un cantón ubicado en la provincia de Sucumbíos al Noroeste del Ecuador, el cual tiene una extensión de 224.208,94 hectáreas, comprendiendo el 12,38\% de la superficie de la provincia de Sucumbíos. "En su división política administrativa se encuentra conformada por la parroquia urbana Lumbaqui y sus parroquias rurales El Reventador, Puerto Libre y Gonzalo Pizarro" (Alvear, 2020, p. 17). El cantón Gonzalo Pizarro se encuentra limitado al norte con el cantón Sucumbíos, al sur el cantón El Chaco de la provincia de Napo, al oeste los cantones 
Pimampiro de la provincia de Imbabura y Cayambe de la provincia de Pichincha, y al este el cantón Cascales de la provincia de Sucumbíos.

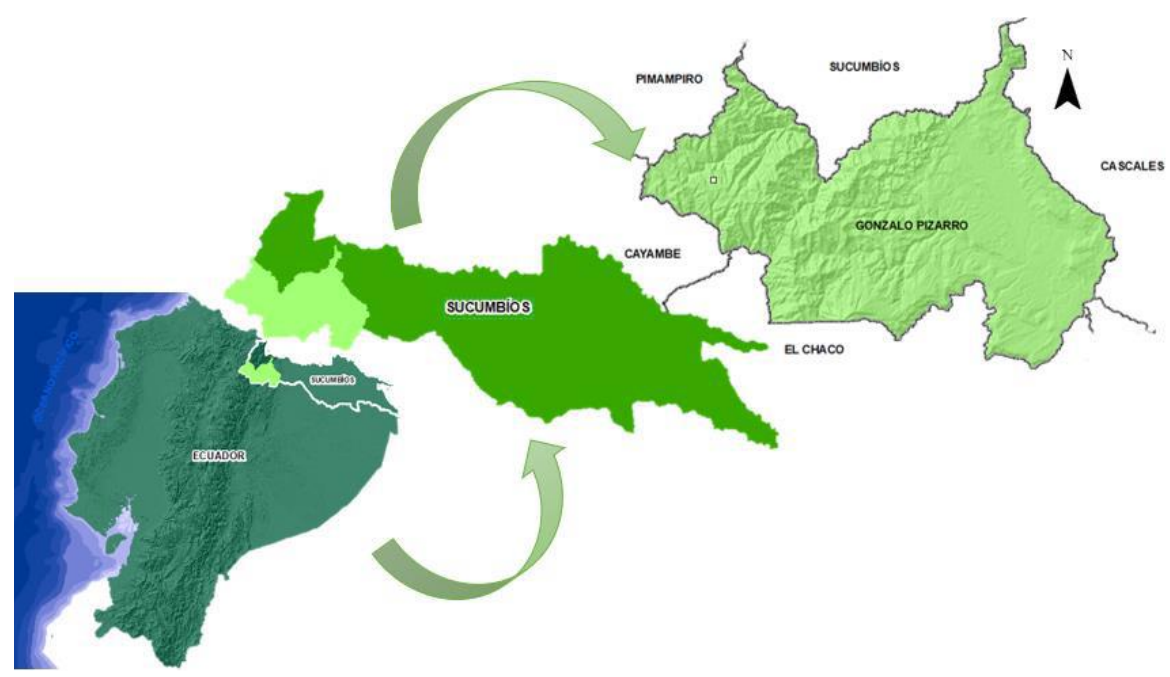

Fuente: (Alvear, 2020)

El Gobierno Autónomo Descentralizado Municipal del cantón Gonzalo Pizarro, dentro de sus competencias exclusivas se encuentra la gestión integral de los desechos sólidos la cual consiste en un conjunto de actividades de recolección, transporte, tratamiento y disposición final de los desechos sólidos. Para garantizar el correcto funcionamiento operacional dispone de dos vehículos recolectores los cuales a través de cuatro rutas brindan el servicio al 89\% del cantón, a la vez cuanta con un relleno sanitario donde se realiza el tratamiento de los desechos sólidos por medio de celdas de disposición final, y el lixiviado generado producto de la descomposición es tratado y una vez que cumple con los límites permisibles es descargado. La cantidad de desechos sólidos que ingresa al relleno sanitario es de 4,5 toneladas al día, la cantidad de material de cobertura utilizado mensualmente es de $35 \mathrm{~m}^{3}$, la cantidad de lixiviado tratado es de $5 \mathrm{~m}^{3}$, la cantidad de lodo generado del tratamiento de lixiviados que es tratado al mes es de $10 \mathrm{~m}^{3}$.

En el Plan de Desarrollo y Ordenamiento Territorial del cantón Gonzalo Pizarro, la gestión integral de los desechos sólidos se encuentra dentro del componente biofísico ambiental donde el objetivo estratégico es

"Propiciar la conservación ambiental, estableciendo como principio la corresponsabilidad ciudadana en el manejo sostenible de los recursos naturales, mediante políticas, normas, instrumentos y acciones de desarrollo que permiten la recuperación de espacios naturales degradados" (Alvear, 2020, p. 91)

Para el cumplimiento se requiere realizar actividades que permitan eliminar los focos de contaminación a través de un manejo adecuado de los desechos sólidos para lo cual es importante el estudio de la capacidad operativa. Partiendo de esta premisa y de la naturaleza del problema la metodología que se adapta a la investigación es de tipo cuantitativa examinando las actividades mediante el estudio de tiempos y movimientos la cual es una herramienta utilizada "para determinar los tiempos estándares de cada una de las operaciones que comprenden cualquier proceso, así como para analizar los movimientos que son realizados por parte de un operario para llevar a cabo dicha operación" (Proaño, et al., 2017, p. 40). Posteriormente se realiza el cálculo de la capacidad operativa a través del análisis de cada uno de los procesos de limpieza y mantenimiento de espacios públicos, recolección y transporte de los desechos sólidos, disposición de los desechos sólidos, y tratamiento de los lixiviados producto de la descomposición de los desechos sólidos.

Para la capacidad total (CT) que comprende el tiempo del operario por puesto de trabajo y depende del ciclo que se encuentra realizando, el cual se lo calcula mediante la siguiente ecuación: 
$C T=\sum_{i=1}^{n} N_{i} * 360 \frac{\text { días }}{\text { año }} 24 \frac{h}{\text { día }}$

Donde

$N \quad$ Número de trabajadores

h Cantidad de horas

La capacidad disponible se define como la diferencia entre la capacidad total y el tiempo no disponible y se lo obtiene de acuerdo a la siguiente ecuación:

$T T D=C T-T N D$

Donde

CT Capacidad Total

TND Tiempo no disponible

La capacidad de operación comprende la diferencia entre el tiempo total disponible y al tiempo no operativo y se lo obtienen con la siguiente ecuación:

$T O=T T D-T N O$

Donde:

TTD Tiempo total disponible

TND Tiempo no operativo

La capacidad de producción comprende la diferencia entre la capacidad de operación y el tiempo no productivo y se lo define con la siguiente ecuación:

TO Tiempo de operación

TNP Tiempo no productivo

La capacidad de funcionamiento comprende la diferencia entre el tiempo de operación y el tiempo de no funcionamiento que comprende interrupciones aleatorias y fortuitas como son daños, averías de máquinas, accidentes de trabajo, y falta repentina del servicio de recolección de desechos sólidos y se lo obtienen con la siguiente ecuación:

$T F=T P-T N F$

Donde

TP Tiempo de producción 
TNF Tiempo de no funcionamiento (interrupciones inesperadas)

La capacidad real se define como la diferencia entre el tiempo de funcionamiento y el tiempo de ajuste comprendido como las interrupciones de todo tipo, pero muy pequeñas siendo difícil su medición. A estas interrupciones se adiciona el tiempo empleado en el mal manejo de los desechos sólidos o mal tratamiento.

\section{RESULTADOS}

Tabla 1 Descripción de actividades de la gestión integral de los desechos sólidos

\begin{tabular}{|c|c|c|c|}
\hline MACRO PROCESO & PROCESO & DESCRIPCIÓN DE ACTIVIDAD & $\begin{array}{l}\text { TIEMPO } \\
\text { (min) }\end{array}$ \\
\hline TRANSPORTE & $\begin{array}{l}\text { Recolección de los } \\
\text { desechos sólidos en las } \\
\text { diferentes rutas }\end{array}$ & $\begin{array}{l}\text { Recorrido de recolección de los desechos en la } \\
\text { ruta Lumbaqui - Amazonas }\end{array}$ & 240 \\
\hline TRANSPORTE & $\begin{array}{l}\text { Recolección de los } \\
\text { desechos sólidos en las } \\
\text { diferentes rutas }\end{array}$ & $\begin{array}{l}\text { Recorrido de recolección de desechos sólidos de } \\
\text { la ruta Puerto Libre }\end{array}$ & 300 \\
\hline TRANSPORTE & $\begin{array}{l}\text { Recolección de los } \\
\text { desechos sólidos en las } \\
\text { diferentes rutas }\end{array}$ & $\begin{array}{l}\text { Recorrido de la recolección de los desechos } \\
\text { sólidos en la ruta Reventador }\end{array}$ & 360 \\
\hline TRANSPORTE & $\begin{array}{l}\text { Recolección de los } \\
\text { desechos sólidos en las } \\
\text { diferentes rutas }\end{array}$ & $\begin{array}{l}\text { Limpieza, y acondicionamiento de vehículos } \\
\text { recolectores luego del trabajo }\end{array}$ & 120 \\
\hline TRANSPORTE & $\begin{array}{l}\text { Recolección de los } \\
\text { desechos sólidos en las } \\
\text { diferentes rutas }\end{array}$ & Lavado de vehículos recolectores & 60 \\
\hline TRANSPORTE & $\begin{array}{l}\text { Recolección de los } \\
\text { desechos sólidos en las } \\
\text { diferentes rutas }\end{array}$ & $\begin{array}{l}\text { Inspección de vehículo antes de realizar los } \\
\text { trabajos }\end{array}$ & 12 \\
\hline TRATAMIENTO & $\begin{array}{l}\text { Limpieza de parterres y } \\
\text { espacios públicos }\end{array}$ & $\begin{array}{l}\text { Lavado y acondicionamiento de coliseo de } \\
\text { Lumbaqui }\end{array}$ & 360 \\
\hline TRATAMIENTO & $\begin{array}{l}\text { Limpieza de parterres y } \\
\text { espacios públicos }\end{array}$ & Lavado de baños del coliseo de Lumbaqui & 60 \\
\hline TRATAMIENTO & $\begin{array}{l}\text { Limpieza de parterres y } \\
\text { espacios públicos }\end{array}$ & Barrido de coliseo de Lumbaqui & 60 \\
\hline TRATAMIENTO & $\begin{array}{l}\text { Limpieza de parterres y } \\
\text { espacios públicos }\end{array}$ & Lavado de baños del coliseo de Gonzalo Pizarro & 360 \\
\hline TRATAMIENTO & $\begin{array}{l}\text { Limpieza de parterres y } \\
\text { espacios públicos }\end{array}$ & Barrido de coliseo de Gonzalo Pizarro & 60 \\
\hline TRATAMIENTO & $\begin{array}{l}\text { Limpieza de parterres y } \\
\text { espacios públicos }\end{array}$ & Barrido de calles de Gonzalo Pizarro & 240 \\
\hline TRATAMIENTO & $\begin{array}{l}\text { Limpieza de parterres y } \\
\text { espacios públicos }\end{array}$ & Barrido de parterres de Lumbaqui & 960 \\
\hline TRATAMIENTO & $\begin{array}{l}\text { Limpieza de parterres y } \\
\text { espacios públicos }\end{array}$ & Poda de árboles & 1920 \\
\hline TRATAMIENTO & $\begin{array}{l}\text { Limpieza de parterres y } \\
\text { espacios públicos }\end{array}$ & Mantenimiento de parque central & 2400 \\
\hline TRATAMIENTO & $\begin{array}{l}\text { Mantenimiento del } \\
\text { relleno sanitario }\end{array}$ & Limpieza de maleza del relleno sanitario & 360 \\
\hline TRATAMIENTO & Mantenimiento del & Barrido de cubil en el relleno sanitario & 12 \\
\hline
\end{tabular}




\begin{tabular}{|c|c|c|c|}
\hline & relleno sanitario & & \\
\hline TRATAMIENTO & $\begin{array}{l}\text { Mantenimiento del } \\
\text { relleno sanitario }\end{array}$ & Limpieza de baños del relleno sanitario & 18 \\
\hline TRATAMIENTO & $\begin{array}{l}\text { Mantenimiento del } \\
\text { relleno sanitario }\end{array}$ & $\begin{array}{l}\text { Limpieza de canaletas de las celdas de } \\
\text { disposición final cerradas }\end{array}$ & 480 \\
\hline TRATAMIENTO & $\begin{array}{l}\text { Mantenimiento del } \\
\text { relleno sanitario }\end{array}$ & $\begin{array}{l}\text { Lavado de tanques del sistema de tratamiento de } \\
\text { lixiviados }\end{array}$ & 720 \\
\hline DISPOSICIÓN FINAL & $\begin{array}{l}\text { Manejo de los desechos } \\
\text { sólidos }\end{array}$ & Pesaje de vehículos recolectores & 6 \\
\hline DISPOSICIÓN FINAL & $\begin{array}{l}\text { Manejo de los desechos } \\
\text { sólidos }\end{array}$ & $\begin{array}{l}\text { Vaciado de desechos sólidos en la celda de } \\
\text { disposición final }\end{array}$ & 60 \\
\hline DISPOSICIÓN FINAL & $\begin{array}{l}\text { Manejo de los desechos } \\
\text { sólidos }\end{array}$ & Conformación de la celda diaria & 180 \\
\hline DISPOSICIÓN FINAL & $\begin{array}{l}\text { Manejo de los desechos } \\
\text { sólidos }\end{array}$ & $\begin{array}{l}\text { Llenado de tanques con lixiviado en el sistema de } \\
\text { tratamiento }\end{array}$ & 120 \\
\hline DISPOSICIÓN FINAL & $\begin{array}{l}\text { Manejo de los desechos } \\
\text { sólidos }\end{array}$ & $\begin{array}{l}\text { Adicionamiento de insumos químicos en el } \\
\text { tratamiento }\end{array}$ & 18 \\
\hline DISPOSICIÓN FINAL & $\begin{array}{l}\text { Manejo de los desechos } \\
\text { sólidos }\end{array}$ & Mezcla y tratamiento de los lixiviados & 120 \\
\hline DISPOSICIÓN FINAL & $\begin{array}{l}\text { Manejo de los desechos } \\
\text { sólidos }\end{array}$ & Control de calidad del lixiviado & 6 \\
\hline DISPOSICIÓN FINAL & $\begin{array}{l}\text { Manejo de los desechos } \\
\text { sólidos }\end{array}$ & Descarga del lixiviado tratado & 30 \\
\hline DISPOSICIÓN FINAL & $\begin{array}{l}\text { Manejo de los desechos } \\
\text { sólidos }\end{array}$ & Vaciado de lodo del sistema de tratamiento & 60 \\
\hline DISPOSICIÓN FINAL & $\begin{array}{l}\text { Manejo de los desechos } \\
\text { sólidos }\end{array}$ & Limpieza de tanques del sistema de tratamiento & 120 \\
\hline ADMINISTRATIVO & $\begin{array}{l}\text { Plan de Manejo } \\
\text { Ambiental }\end{array}$ & Elaboración de registros & 3 \\
\hline ADMINISTRATIVO & $\begin{array}{l}\text { Plan de Manejo } \\
\text { Ambiental }\end{array}$ & Realización de inspecciones periódicas & 18 \\
\hline ADMINISTRATIVO & $\begin{array}{l}\text { Plan de Manejo } \\
\text { Ambiental }\end{array}$ & Elaboración de informes técnicos & 180 \\
\hline ADMINISTRATIVO & $\begin{array}{l}\text { Plan de Manejo } \\
\text { Ambiental }\end{array}$ & Socialización de la ordenanza & 6 \\
\hline ADMINISTRATIVO & $\begin{array}{l}\text { Plan de Manejo } \\
\text { Ambiental }\end{array}$ & $\begin{array}{l}\text { Elaboración del Plan de Gestión Integral de los } \\
\text { Desechos Sólidos }\end{array}$ & 120 \\
\hline TIEMPO TOTAL (min) & & & 10149 \\
\hline TIEMPO TOTAL (h) & & & 169,15 \\
\hline
\end{tabular}

Fuente: (Morales, 2022)

En la tabla 1 se describe cada una de las actividades desarrolladas en los procesos de limpieza y mantenimiento de espacios públicos, recolección y transporte de los desechos sólidos, disposición de los desechos sólidos, y tratamiento de los lixiviados producto de la descomposición de los desechos sólidos, donde se determina el tiempo destinado para cada tarea, siendo el total de 169,15 horas.

Tabla 2 Capacidad operativa de la gestión integral de los desechos sólidos

\begin{tabular}{lcc}
\hline PARÁMETRO & VALOR & UNIDAD \\
\hline CAPACIDAD TOTAL $($ CT) & 61740 & $\mathrm{~h} / \mathrm{año}$ \\
\hline TIEMPO NO DISPONIBLE $($ TND) & 616 & $\mathrm{~h} / \mathrm{año}$ \\
\hline
\end{tabular}




\begin{tabular}{lcc}
\hline CAPACIDAD DISPONIBLE (CD) & 61124 & $\mathrm{~h} / \mathrm{año}$ \\
\hline TIEMPO DE NO PRODUCCIÓN (TNP) & 2190 & $\mathrm{~h} / \mathrm{año}$ \\
\hline TIEMPO DE PRODUCCIÓN (TP) & 58934 & $\mathrm{~h} / \mathrm{año}$ \\
\hline TIEMPO DE NO FUNCIONAMIENTO (TNF) & 24 & $\mathrm{~h} / \mathrm{año}$ \\
\hline CAPACIDAD DE FUNCIONAMIENTO (TF) & 58910 & $\mathrm{~h} / \mathrm{año}$ \\
\hline TIEMPO DE AJUSTE (TA) & 48 & $\mathrm{~h} / \mathrm{año}$ \\
\hline CAPACIDAD REAL (CR) & 58862 & $\mathrm{~h} / \mathrm{año}$ \\
\hline EFICIENCIA (EF) & 65,21 & $\%$ \\
\hline CANTIDAD DE TRABAJADORES CALCULADO & 23 & Trab. \\
\hline CANTIDAD REAL DE TRABAJADORES & 16 & Trab.
\end{tabular}

Fuente: (Morales, 2022)

En la tabla 2 se describe cada uno de los parámetros para el cálculo de la capacidad operativa, siendo la capacidad total de 61740 h/año la capacidad real es de 58862 h/año, con una cantidad de trabajadores de 23, pero en realidad para realizar las actividades de la gestión integral de los desechos sólidos es de 16 trabajadores motivo por el cual la eficiencia en cuanto al cumplimiento de los objetivos es de $65,21 \%$.

Tabla 3 Recurso necesario para operatividad

\begin{tabular}{lcc}
\hline PARÁMETRO & VALOR & UNIDAD \\
\hline Presupuesto calculado para operatividad & $551.831,25$ & \$USD \\
\hline Presupuesto real & $456.698,88$ & \$USD \\
\hline Brecha & $95.132,37$ & \$USD \\
\hline
\end{tabular}

Fuente: (Morales, 2022)

En la tabla 3 se observa el presupuesto necesario para realizar la gestión integral de los desechos sólidos con respecto al valor real designado para realizar las diferentes actividades de limpieza y mantenimiento de espacios públicos, recolección y transporte de los desechos sólidos, disposición de los desechos sólidos, y tratamiento de los lixiviados producto de la descomposición de los desechos sólidos.

\section{CONCLUSIONES}

De conformidad al análisis realizado se tiene que la capacidad real de la gestión integral de los desechos sólidos es de $58.862 \mathrm{~h} / \mathrm{año}$, teniendo una eficiencia de $65,21 \%$, esto es debido a variables de causas asignables que se debe tomar en cuenta (trabajos múltiples, paradas continuas de maquinaria por mantenimiento correctivo, actividades operativas imprevistas), que no tienen nada que ver con la operatividad y la planificación.

De conformidad al artículo 565 del Reglamento del Código Orgánico Ambiental, el mismo que lleva concordancia con el artículo 431 del Código Orgánico de Ordenamiento Territorial, Autonomía y Descentralización, se concluye que las actividades operativas han sido realizadas tomando en cuenta la capacidad real y la disminución en cuanto al rendimiento se debe a la falta de una planificación de los mantenimientos.

Después de realizar el análisis del involucramiento en el desarrollo de un documento técnico para ser aplicado en la gestión integral de los desechos sólidos, nuestro modelo de planificación de mantenimiento se aplica considerando la realidad local y es válido para evidenciar el cumplimiento de los requerimientos legales. 


\section{Referencias}

Alvear, C., 2020. Plan de Desarrollo y Ordenamiento Territorial del cantón Gonzalo Pizarro. 1 ed. s.1.:GAD Gonzalo Pizarro.

Bejarano, et. al., 2016. Diseño del modelo para la planeación de capacidad operativa de la empresa GMT. 1 ed. s.1.:Pontificia Universidad Javeriana.

Carabalí, M., 2016. Evaluar la capacidad operativa del área de almacenamiento de residuos ordinarios de Calima Centro Comercial mediante la gestión de los riesgos, siguiendo los lineamientos planteados por el PMBOK. Universidad Militar Nueva Granada, 1(1), pp. 1 - 26.

Cassaretto, et. al., 2016. Modernización de la cadena de suministros de combustibles de aviación y su impacto en la capacidad operativa de las Unidades Áreas FAP. 1 ed. s.l.:Universidad ESAN.

Clavijo \& López, 2015. Capacidad operativa de las empresas de transporte de pasajeros en Ocaña y su región. 1 ed. s.l.:Universidad Francisco de Paula Santander Ocaña.

Córdova, S., 2019. La administración de operaciones y su papel central en la organización. 1 ed. s.l.s.n.

Hernández, et. al., 2017. Análisis de las capacidades operativas en empresas manufactureras. Revista Internacional Administración y Finanzas, 10(6), pp. 17 - 28.

Hernández, et. al., 2017. Análisis de las capacidades operativas en empresas manufactureras. Revista Internacional Administración \& Finanzas, 10(6), pp. 17 - 28.

Máynez \& Cavazos, 2013. Capacidad operativa para innovar y ventaja competitiva: análisis desde la percepción de actores clave en el sector productivo fronterizo. p. 22.

Montes, N., 2014. Criterios para determinar capacidad de producción en empresas de transformación PYMES. s.l.:Universidad Autónoma de México.

Proaño, D., Soler, V. \& Pérez, E., 2017. Metodología de estudio de tiempo y movimiento; introducción al GSD. 3C Empresa Investigación y Pensamiento Crítico, Edición Especial(1), pp. 39 - 49.

Ritala \& Blomqvist, 2009. Innovación orchestration capability - defining the organizational and individual level determinants. International Journal of Innovation Management, 13(4), pp. 569 - 591. 